\title{
ALGEBRAIC POSTULATES AND A GEOMETRIC INTER- PRETATION FOR THE LEWIS CALCULUS OF STRICT IMPLICATION
}

\author{
TANG TSAO-CHEN
}

1. Two further postulates for a Boolean ring with a unit element. If addition, subtraction, and multiplication are properly defined in logic, it may be shown* that the postulates for these operations are identical with those in a ring, in which every element is idempotent, satisfying the postulate $x x=x$. Such a ring is called a Boolean ring. The postulates for a Boolean ring with a unit element are therefore the following:

A. Addition is always possible, commutative, and associative.

B. Multiplication is always possible, associative, and both left-and right-distributive with respect to addition.

C. Subtraction is always possible.

D. $x x=x$.

E. There exists an element 1 such that $x 1=x$ for every element $x$ in the ring.

Here we shall introduce a new operation, represented by $x^{\infty}$, which satisfies the following two further postulates:

$\mathrm{F}_{1}$. For every element $x$ there exists an element $x^{\infty}$ such that $x^{\infty} x=x^{\infty}$.

$\mathrm{F}_{2}$. For any two elements $x$ and $y$ we have $(x y)^{\infty}=x^{\infty} y^{\infty}$.

The postulates $\mathrm{A}-\mathrm{F}_{2}$, obtained above, may be called the algebraic postulates for the Lewis calculus of strict implication.

2. A geometric meaning of the symbol $x^{\infty}$. A geometric meaning $\dagger$ may be attached to $x^{\infty}$ as follows : Let $x$ be a point set in the euclidean

* See M. H. Stone. The theory of representations for Boolean algebras, Transactions of this Society, vol. 40 (1936), pp. 37-53.

$\dagger$ Another geometric meaning of $x^{\infty}$ may be obtained by assuming $1^{\infty}$ to be any one fixed point or any set of fixed points (finite or infinite in number and continuous or discontinuous in character) and setting $x^{\infty}=x 1^{\infty}$. If we assume that $1^{\infty}$ is a fixed point, we have then the following property:

G. $x^{\infty}$ is two-valued, that is, $x^{\infty}=1^{\infty}$ or $0^{\infty}$,

which is independent of the postulates $A-F_{2}$. This sub-Boolean algebra with the postulates A-G does not become the ordinary two-valued Boolean algebra, unless we assume further that $x$ is two-valued. 
plane; then $x^{\infty}$ may be taken as the interior points of $x$ (the set of all points $p$ such that some circle with center at $p$ lies entirely within $x$ ). Then the postulates $\mathrm{F}_{1}$ and $\mathrm{F}_{2}$ are satisfied. Moreover $(1-x)^{\infty}$ is the set of points interior to the complement of $x$, that is, exterior of $x$; $1-x^{\infty}-(1-x)^{\infty}$ is the frontier of $x$; and $1-(1-x)^{\infty}$ is the closure of $x$, that is, $x$ plus its frontier.

3. A geometric interpretation of Lewis' possibility functions. When $p$ is a proposition, Lewis introduces an undefined idea $\diamond p$, which is read " $p$ is possible" and may be called the possibility function of $p$. Now, if a class $x$ be given, $\diamond x$ must have a corresponding meaning which is obtained from the following definition:

DEFINITION. $\diamond x=1-(1-x)^{\infty}$.

By means of the definition of $\sim x$, we obtain $\sim x=1-x$; and we have the following theorems:

THEOREM 1. $\diamond x$ is the closure of $x$.

THEOREM 2. $\sim \diamond x=(1-x)^{\infty}$ is the exterior of $x$.

THEOREM 3. $\diamond \sim x=1-x^{\infty}$ is the closure of the complement of $x$.

THEOREM 4. $\sim \diamond \sim x=x^{\infty}$ is the interior of $x$.

On the basis of the postulates A-F we may then prove abstractly the following theorems:

THEOREM $5.0^{\infty}=0$ and $\sim \diamond \sim 0 .=.0$; that is, the interior of the null class is a null class.

TheOREM 6. $\sim \diamond \sim x . \sim \diamond x:=.0$, and $x^{\infty}(\sim x)^{\infty}=0$; that is, if $x$ is a class, then the interior and the exterior of $x$ have no point in common.

Definition. $x^{f}=1-x^{\infty}-(\sim x)^{\infty}$, that $i s, x^{f}$ is the frontier of $x$.

THEOREM 7. $x^{f}=(\sim x)^{f}$, that is, $x$ and $\sim x$ have the same frontier.

ThEOREM 8. $x^{f}+x^{\infty}+(\sim x)^{\infty}=1, x^{f}+(\sim \diamond \sim x)+(\sim \diamond x)=1$; that is, the frontier, the interior, and the exterior of $x$ form the whole plane.

Theorem 9. $\diamond x .=. x^{f}+x^{\infty}$ and $\diamond x .=. x^{f}+(\sim \diamond \sim x)$; that is, $\diamond x$ is the sum of the frontier and the interior of $x$.

THEOREM 10. $\diamond \sim x .=. x^{f}+(\sim x)^{\infty}$ and $\diamond \sim x .=. x^{f}+\sim \diamond x$; that $i s, \diamond \sim x$ is the sum of the frontier and the exterior of $x$.

Theorem 11. $x(\sim x)^{\infty}=0$ and $x . \sim \diamond x:=.0$; that is, the class $x$ and its exterior have no point in common. 
THEOREM 12. $x=x^{\infty}+x x^{f}$ and $x=(\sim \diamond \sim x)+x x^{f}$; that is, the class $x$ is the sum of its interior and the part of its frontier which belongs to it.

THEOREM 13. $(\sim x)=(\sim x)^{\infty}+(\sim x) x^{f}$ and $(\sim x)=(\sim \diamond x)+(\sim x) x^{f}$; that is, the class $\sim x$ is the sum of the exterior of $x$ and the part of its frontier which belongs to $\sim x$.

4. Theorems corresponding to Lewis' postulates. In Symbolic Logic by Lewis and Langford the symbol $p-3 q$ is defined by $\mathrm{p}-3 q .=. \sim \diamond(p \sim q)$. Changing the propositions $p$ and $q$ into the classes $x$ and $y$, we get the definition of $x-3 y$ :

Definition. $x-3 y_{0}=\sim \sim \diamond(x \sim y)$.

The geometric meaning of $x-3 y$ is very clear, for its definition states that $x-3 y$ means the exterior of $x \sim y$; that is, if, for example, two curves $x^{c}$ and $y^{c}$ divide the plane into four parts $x y, \sim x y, x \sim y$, and $\sim x \sim y$, as in the figure, then $x-3 y$ means the interior of $x>y$, which is the sum of $x y, \sim x y$, and $\sim x \sim y$. In other words, we have the following two theorems:

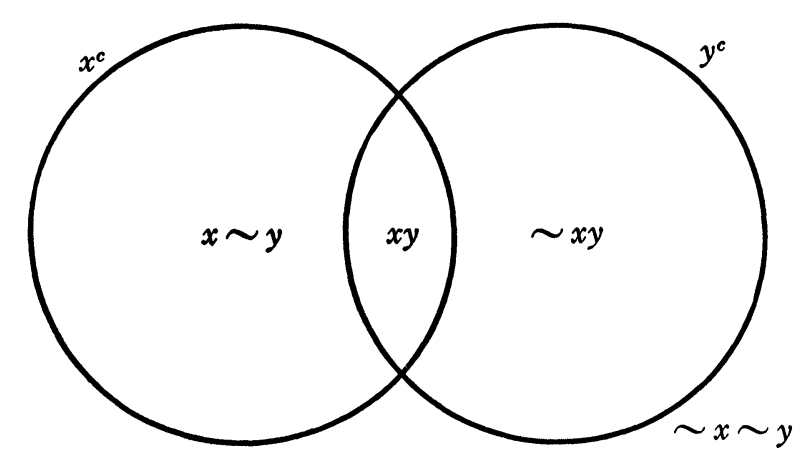

Theorem 14. $x \supset y .=. x y+(\sim x y)+(\sim x \sim y) . *$

Theorem 15. $x-3 y .=. \sim \diamond \sim(x \supset y) .=.(x \supset y)^{\infty}$.

Proof. By the definition of $\sim x, \sim x .=.1-x$, and we see that

$$
\begin{aligned}
x y+(\sim x y)+(\sim x \sim y) & =x y+(1-x) y+(1-x)(1-y) \\
& =x y+y-x y+1-x-y+x y \\
& =1-x+x y \\
& =(x \supset y) .
\end{aligned}
$$

* $x \supset y$ is, of course, defined as $\sim(x \sim y)$ which is $1-x+x y$. 
By Theorem 4

DEFINITION. $i=(\sim \diamond 0)$.

$$
\begin{aligned}
\sim \diamond \sim(x \supset y) . & =.(x \supset y)^{\infty}, \\
\sim \diamond \sim(x \supset y) . & =\sim \diamond \sim(1-x+x y) \\
& =. \sim \diamond[1-(1-x+x y)] \\
& =. \sim \diamond(x-x y) \\
& =. \sim \diamond x(1-y) \\
& =. \sim \diamond(x \sim y) \\
& =. x-3 y .
\end{aligned}
$$

This definition of $i$ may be replaced by the following theorem:

THEOREM 16. $i=1^{\infty}$ and $i=(\sim \diamond \sim 1)$.

Proof. We have $i=(\sim \diamond 0)=[\sim \diamond(1-1)]=(\sim \diamond \sim 1)=1^{\infty}$, by Theorem 3.

THEOREM 17. $x-3 x .=. i$.

Proof. We have $x-3 x .=. \sim \diamond(x \sim x)=. \sim \diamond 0=. i$.

If $p$ and $q$ are propositions, then we have the following theorem:* If $p-3 q$ is asserted, then $p-3 q .=. i$.

Lewis' postulates may therefore be written as follows:

$$
\begin{aligned}
& p q-3 q p .=. i, \\
& p q-3 p .=. i, \\
& p-3 p p .=. i, \\
&(p q) r-3 p(q r) .=. i, \\
& p-3 \sim(\sim p) .=. i, \\
& p-3 q . q-3 r:-3 . p-3 r:=. i \\
& p \cdot p-3 q:-3 . q: .=. i \\
& \diamond(p q)-3 \diamond p .=. i
\end{aligned}
$$

The corresponding theorems for classes are obtained by changing the propositions $p, q, r$ into $x, y, z$, respectively:

THEOREM 18. $x y-3 y x .=. i$.

Proof. We have $x y-3 y x .=. x y-3 x y=. i$ by Theorem 17.

\footnotetext{
* See this Bulletin, vol. 42 (1936), p. 708, Theorem 9.
} 
Theorem 19. $x y-3 x .=. i$.

Proof. We have $x y-3 x .=. \sim \diamond(x y \sim x)=. \sim \diamond(y 0)=. \sim \diamond 0=. i$.

In like manner we may prove Theorems 20-22 below:

THEOREM 20. $x-3 x x .=. i$.

THEOREM 21. $(x y) z-3 x(y z) .=. i$.

Theorem 22. $x-3 \sim(\sim x) .=. i$.

Theorem 23. $x-3 y \cdot y-3 z:-3 \cdot x-3 z: .=. i$.

Proof. We have

$$
\begin{aligned}
x-3 y \cdot y-3 z:-3 \cdot x-3 z: & =:(x \supset y)^{\infty}(y \supset z)^{\infty} \cdot-3 \cdot x-3 z \\
& =:(x \supset y \cdot y \supset z)^{\infty} \cdot-3 \cdot x-3 z \\
& =:(x \supset y \cdot y \supset z \cdot x \supset z)^{\infty} \cdot-3 \cdot x-3 z \\
& =:(x \supset y)^{\infty}(y \supset z)^{\infty}(x \supset z)^{\infty} \cdot-3 \cdot x-3 z \\
& =: x-3 y \cdot y-3 z \cdot x-3 z:-3 \cdot x-3 z \\
& =: x-3 z \cdot x-3 y \cdot y-3 z \cdot:-3 \cdot x-3 z \\
& =: i,
\end{aligned}
$$

where the first five equations can be obtained, respectively, from Theorem 15, $\mathrm{F}_{2}$, Theorem 23.1 (below), $\mathrm{F}_{2}$, and Theorems 4 and 15. The last equation follows from Theorem 19.

THEOREM 23.1. $x \supset y \cdot y \supset z:=: x \supset y \cdot y \supset z . x \supset z$.

Proof. We have

$$
\begin{aligned}
x \supset y . y \supset z: & =:(\sim x+x y) .(\sim y+y z) \\
& : \sim \sim x \sim y+\sim x y z+x y \sim y+x y y z \\
& :: \sim x \sim y+\sim x y z+0+x y z \\
& :: \sim x \sim y+y z .
\end{aligned}
$$

Then

$$
\begin{aligned}
x \supset y . y \supset z . x \supset z .: & =:(\sim x \sim y+y z) .(x \supset z) \\
= & :(\sim x \sim y+y z) .(\sim x+x z) \\
= & : \sim x \sim x \sim y+\sim x y z+0+x y z z \\
= & : \sim x \sim y+\sim x y z+x y z \\
= & : \sim x \sim y+y z \\
& =: x \supset y . y \supset z,
\end{aligned}
$$


by (1) above.

Theorem 24. $x \cdot x-3 y:-3 \cdot y: .=. i$.

Proof. We have

$$
\begin{aligned}
x . x-3 y:-3 . y: . & =. \sim \diamond(x . x-3 y \cdot \sim y) \\
& =. \sim \diamond(x \sim y \cdot x-3 y) \\
& =. \sim \diamond(x \sim y \cdot \sim \diamond(x \sim y)) \\
& =. \sim \diamond 0 \\
& =. i
\end{aligned}
$$

by Theorem 11 and a definition.

THEOREM 25. $\diamond(x y)-3 \diamond x .=. i$.

Proof. By the application of Theorem 25.1 (below),

$$
\begin{aligned}
\diamond(x y)-3 \diamond x . & =. \sim \diamond[\diamond(x y) \sim \diamond x] \\
& =. \sim \diamond[\diamond(x y) \sim \diamond x \sim \diamond(x y)] \\
& =. \sim \diamond(\sim \diamond x .0) \\
& =. \sim \diamond 0 \\
& =. i
\end{aligned}
$$

by definition.

THEOREM 25.1. $\sim \diamond x .=: \sim \diamond x . \sim \diamond(x y)$.

Proof. We have, using $\mathrm{F}_{2}$,

$$
\begin{aligned}
\sim \diamond x . \sim \diamond(x y): & =.(1-x)^{\infty}(1-x y)^{\infty}, \\
& =\cdot[(1-x)(1-x y)]^{\infty} \\
& =.(1-x-x y+x x y)^{\infty} \\
& =.(1-x)^{\infty} \\
& =. \sim \diamond x,
\end{aligned}
$$

where the first step follows by Theorem 1 and the last by Theorem 2 .

5. Theorems corresponding to Lewis' operations. Lewis' substitution can be performed both on propositions and on classes. We consider therefore only adjunction and inference. For this purpose, we first establish the following two theorems:

THEOREM 26. $x \cdot x-3 y:=: x y \cdot x-3 y$. 
Proof. By Theorem 11 we have

$$
x \sim y . \sim \diamond(x \sim y):=.0,
$$

or

$$
x(1-y) \cdot x-y:=.0,
$$

or

$$
(x-x y) \cdot x-3 y:=.0,
$$

or

$$
(x \cdot x-3 y)-(x y \cdot x-3 y)=0,
$$

from which we get at once Theorem 26.

Theorem 27. (1) If $i-3 x .=. i$, then $i x=i$. (2) Conversely, if $i x=i$, then $i-3 x .=. i$.

Proof. We obtain, using Theorem 16, $\mathrm{F}_{2}$, and Theorem 26, $i x=1^{\infty} x=(1.1)^{\infty} x=1^{\infty} 1^{\infty} x=i i x=i x i=i x(i-3 x)=i(i-3 x)=i i=i$.

The proof of the converse theorem is the following: We have $i-3 x .=. \sim \diamond(i \sim x)=. \sim \diamond[i \sim(i x)]=. \sim \diamond(i \sim i)=. \sim \diamond 0=. i$, by definition.

DEFINITION. We write $\vdash x$, when and only when $i x=i$.

Thus, we may write $\vdash x$, when $x=1$ or $x=i$. For example, Theorem 18 is equivalent to $\vdash . x y-3 y x$.

We can now prove the theorems corresponding to adjunction and inference.

THEOREM 28. If $\vdash x$ and $\vdash y$, then $\vdash(x y)$.

Proof. We have $i(x y)=i i x y=i x i y=i i=i$. Hence by definition, $\vdash(x y)$.

THEOREM 29. If $\vdash x$, and $\vdash . x-3 y$, then $\vdash y$.

Proof. We obtain $i y=i i y=(i x)(i \cdot x-3 y) y=i x i(x-3 y) y=i i x y(x-3 y)$ $=i i x(x-3 y)=(i x) i(x-3 y)=i i=i$, using Theorem 26. Therefore $i y=i$; hence by definition $\vdash y$.

6. Miscellaneous theorems. We state the following theorems, each without proof :

THEOREM 30. $x-3 y .=: i . x-3 y$.

THEOREM 31. If $x-3 y .=. i$, and $y-3 z .=. i$, then $x-3 z .=. i$. 
THEOREM 32. If $x-3 y .=. i$ and $x-3 y .-3 \cdot u-3 v:=. i$, then $u-3 v$. $=. i$.

Theorem 33. (1) If $x-3 y .=. i$, then $\vdash . x-3 y$. (2) Conversely, if $\vdash . x-3 y$, then $x-3 y .=. i$.

Theorem 34. (1) If $i-3 x .=. i$, then $\vdash x$. (2) Conversely, if $\vdash x$, then $i-3 x .=. i$.

TheOREM 35. If $1-3 x .=. i$, then $\vdash x$.

7. A relation between Huntington's " $p$ in $T$ " and " $\vdash p$ " in this paper. In this Bulletin (vol. 40 (1934), p. 733) E. V. Huntington established the theorem

$$
(p<q) \rightleftarrows[(p-3 q) \text { in } T]
$$

from the following definition of $(p<q)$ :

$$
(p<q) \rightleftarrows(p=p q) \text {. }
$$

It is now my aim to prove the following theorem:

TheOREM 36. $[(p-3 q)$ in $T] \rightleftarrows(\vdash \cdot p-3 q)$.

Proof of $[(p-3 q)$ in $T] \rightarrow(\vdash \cdot p-3 q)$. By hypothesis, $(p-3 q)$ in $T$. By (2) and (3), $p=p q$. Therefore, $p-3 q .=. p q-3 q .=. i$. Thus $\vdash \cdot p-3 q$.

Proof of $(\vdash \cdot p-3 q) \rightarrow[(p-3 q)$ in $T]$. By (2) and (3) $(p=p p)$ $\rightarrow[(p-3 p)$ in $T]$. Therefore, $(p-3 p)$ in $T$, that is,

$$
i \text { in } T \text {. }
$$

But by hypothesis, $i . p-3 q:=. i$. Hence

$$
p-3 q .=. i \text {. }
$$

From (4) and (5) we have at once $(p-3 q)$ in $T$.

National WU-Han University 